\title{
Angina Cranialis: An Unusual Presentation of Referred Ischemic Myocardial Pain
}

\author{
Pornchai Sathirapanya
}

\begin{abstract}
Headache was a very unusual presentation of acute myocardial ischemia. A case of acute myocardial infarction presented initially with only excruciating headache was reported. The abnormal electrocardiography and high level of serum creatine kinase-cardiac type (CK-MB) and cardiac troponin T confirmed the diagnosis. The headache totally disappeared after the intensive medical treatment of ischemic heart disease. This rare presentation of acute myocardial infarction should be considered in acutely severe headache cases after the intracranial causes of headache have been completely excluded.
\end{abstract}

Keywords: Acute myocardial infarction; Creatine kinase-cardiac type (CK-MB); Cardiac troponin T; Non-exertional headache

\section{Introduction}

Headache as a sole initial presentation of acute myocardial infarction was found less than $6 \%$ [1]. Though exertional type of headache has been reported in the majority of cases, non-exertional type of headache caused by acute myocardial infarction has also been presented [2]. The paucity of such cases, fewer associated typical cardiac symptoms and the non-exertional type of headache frequently caused misdiagnosis.

\section{Case Report}

A 72-year-old previously healthy male presented with acute dull-aching headache on his right occipital scalp with radi-

Manuscript accepted for publication October 1, 2012

Division of Neurology, Department of Internal Medicine, Faculty of Medicine, Prince of Songkla University, Hat Yai, Songkhla 90110 Thailand.Email: sporncha@medicine.psu.ac.th

doi: http://dx.doi.org/10.4021/jnr141w ating pain to his right shoulder shortly after wakening ten days before his presentation. This headache spontaneously resolved without any treatment. A few days later, he had another abrupt, seriously intense and similar headache accompanied by palpitation, sweating and nausea. He was immediately presented to the emergency department of a local hospital where no abnormality in neurological examination was found. An emergency computed tomographic brain scan and a lumbar puncture were performed to exclude subarachnoid hemorrhage. Both of the investigations were unremarkable. Thereafter, he was transferred to the author's center for further evaluation and management of this unresolved headache.

On initial evaluation the patient had only tachycardia but normal blood pressure and body temperature. Repeated neurological examination was normal and no meningeal irritation signs were evidenced. A baseline electrocardiography (ECG) showed a prominent Q wave in V1-V4 in association with ST segments elevation and inverted T waves in V1-V5. The immediate cardiac enzymes tests showed total serum creatine kinase-cardiac type (CK-MB) was $246.2 \mathrm{ng} / \mathrm{mL}$ and cardiac troponin T was $6.1 \mathrm{ng} / \mathrm{mL}$. A transthoracic echocardiogram revealed faired left ventricular systolic function and septal and apical myocardial wall akinesia with a left ventricular ejection fraction of $40 \%$. Neither intracardiac clot nor pericardial effusion was detected. On cardiovascular risk factors screening, he had normal fasting blood sugar, lipid profiles and renal function tests. A long history of smoking was the only notable cardiovascular risk factor reported. Thus, acute anterior wall myocardial infarction was the diagnosis. The headache totally disappeared over a few days after intensive medical treatments for acute cardiac ischemia with isosorbide dinitrate, beta blocker and antiplatelet. On the third day of admission, he developed acute pulmonary edema followed by ischemic stroke. Both of the complications were attributed to low cardiac output from left ventricular dysfunction and responded well to treatments. He refused to undergo cardiac angiography and interventional treatment. He has been free from cardiac symptoms for seven years till now with regular anti-ischemic heart disease treatment. It was a matter of interest for the diagnosis that there was no any chest pain and non-exertional headache was merely a 


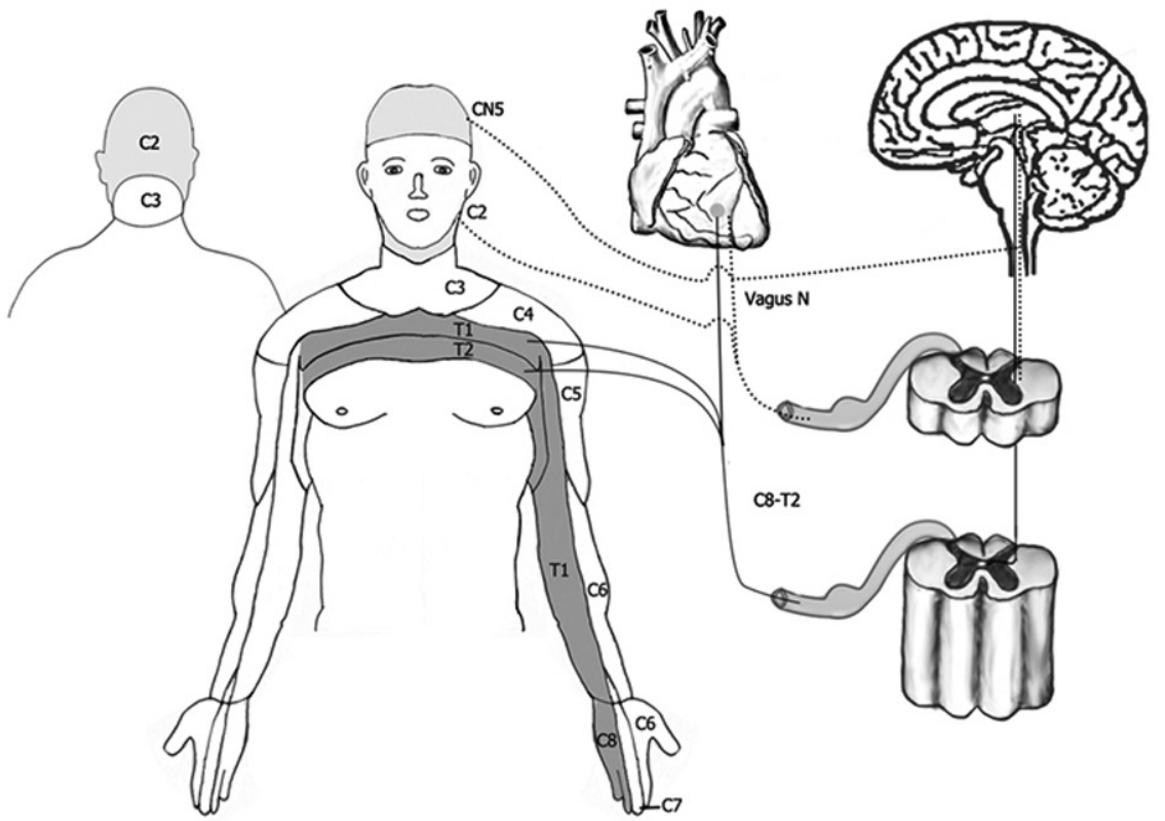

Figure 1. Usual (solid line) and overflow of usual neural convergence (dotted line) of referred pain from myocardial ischemia.

dominant presenting symptom of acute myocardial infarction in this patient.

\section{Discussion}

The pathogenesis of headache caused by myocardial ischemia has remained inconclusive. The convergence of somatic inputs ( $\mathrm{C} 8$ to $\mathrm{T} 2$ dermatome) with central autonomic pathways at the thalamus or with peripheral autonomic afferents which conveys the cardiac pain at the spinal cord was proposed as the usual mechanism responsible for cardiac referred pain [3]. The variations in the locations of cardiac referred pain were therefore attributed to the variations in these somatic convergences, either ascent or descent of the neural convergence between the somatic sensory fibers and the autonomic afferents from the heart; so called "overflow of usual neural convergence" [4]. For example, the referred cardiac pain can occur at vertex, orbit, bregma, nasal tip, frontal and peri-oral areas in cases of ascending convergence to involve descending sensory fibers of trigeminal nerve in brain stem as in this reported case, or at epigastrium and peri-umbilical areas in cases of descending neural convergence [5-7] (Fig. 1). Another proposed mechanism was the release of potent cerebral vasodilators from the ischemic myocardium such as serotonin, bradykinin, substance $\mathrm{P}$, histamine or atrial natriuretic factor [3].

A concomitant characteristic abnormality in electrocardiography (ECG) and tachycardia raised a suspicion of a cardiac cause of headache in this case after intracranial hemorrhage was completely excluded. The normal neurological examination including the absence of meningeal irritation signs and normal cerebrospinal fluid analysis strongly excluded intracranial hemorrhages; focusing on subarachnoid hemorrhage. The abnormal baseline ECG, high level of CK$\mathrm{MB}$ and cardiac troponin T confirmed acute myocardial injury. In some instances, when all the initial cardiac evaluations were unremarkable but ischemic heart disease was still highly suspected, an exercise stress test or a coronary angiography was indicated for the definite diagnosis [2].

The differentiation of cardiac causes of headache and from subarachnoid hemorrhage with a typical myocardial ischemic pattern on electrocardiogram (ECG) is actually a clinical challenge. Cautiously complete neurological evaluations can exclude the intracranial causes of severe headache. Computed tomographic scan of brain and cerebrospinal fluid analysis are strongly needed in all cases with the presentation of so-called thunderclap headache to exclude subarachnoid hemorrhage unavoidably first like in this case report. Since subarachnoid hemorrhage is much more common than ischemic heart disease to produce severe headache, intracranial lesion has to be excluded promptly. Both myocardial infarction induced headache and subarachnoid hemorrhage with ischemic ECG pattern reflected a neural correlation between heart and brain possibly via the neural convergence between autonomic and somatic sensory pathways as proposed.

\section{Conclusion}

A case of acute anterior wall myocardial infarction presented with excruciating non-exertional headache was presented. Though the definite neural pathogenesis has not been elu- 
cidated, a variation of convergence between cardiac autonomic and somatic sensory afferents either at peripheral or central nervous system was a possible explanation.

\section{Acknowledgement}

The author thanks Jude Hall and David Patterson of International Affairs Unit, Faculty of Medicine, Prince of Songkla University for suggestions and editing of English writing. The author thanks Chatchuea Chueakaew for drawing the illustration.

\section{Conflict of Interest and Funding}

No.

\section{Patient's Signs Consent Form}

Obtained.

\section{Ethical Consideration and Registration}

By Ethics Committee of Faculty of Medicine, Prince of
Songkla University.

\section{References}

1. Sampson JJ, Cheitlin MD. Pathophysiology and differential diagnosis of cardiac pain. Prog Cardiovasc Dis. 1971;13(6):507-531.

2. Bini A, Evangelista A, Castellini P, Lambru G, Ferrante T, Manzoni GC, Torelli P. Cardiac cephalgia. J Headache Pain. 2009;10(1):3-9.

3. Meller ST, Gebhart GF. A critical review of the afferent pathways and the potential chemical mediators involved in cardiac pain. Neuroscience. 1992;48(3):501524.

4. Grace A, Horgan J, Breathnach K, Staunton H. Anginal headache and its basis. Cephalalgia. 1997;17(3):195196.

5. Lipton RB, Lowenkopf T, Bajwa ZH, Leckie RS, Ribeiro S, Newman LC, Greenberg MA. Cardiac cephalgia: a treatable form of exertional headache. Neurology. 1997;49(3):813-816.

6. Lefkowitz D, Biller J. Bregmatic headache as a manifestation of myocardial ischemia. Arch Neurol. 1982;39(2):130.

7. Caskey WH, Spierings EL. Headache and heartache. Headache. 1978;18(5):240-243. 